\title{
Forecasts on the speed of gravitational waves at high $z$
}

\author{
Alexander Bonilla ${ }^{a}$, Rocco D’Agostino ${ }^{b, c}$, Rafael C. Nunes ${ }^{d}$, José \\ C. N. de Araujo ${ }^{d}$
}

\author{
${ }^{a}$ Departamento de Física, Universidade Federal de Juiz de Fora, 36036-330, Juiz de Fora, \\ MG, Brazil. \\ ${ }^{b}$ Dipartimento di Fisica, Università di Napoli "Federico II", Via Cinthia 21, I-80126, Napoli, \\ Italy. \\ ${ }^{c}$ Istituto Nazionale di Fisica Nucleare (INFN), Sezione di Napoli, Via Cinthia 9, I-80126 \\ Napoli, Italy. \\ ${ }^{d}$ Divisão de Astrofísica, Instituto Nacional de Pesquisas Espaciais, Avenida dos Astronautas \\ 1758, São José dos Campos, 12227-010, SP, Brazil. \\ E-mail: abonilla@fisica.ufjf.br, rdagostino@na.infn.it,rafadcnunes@gmail.com, \\ jcarlos.dearaujo@inpe.br
}

\begin{abstract}
The observation of GW170817 binary neutron star (BNS) merger event has imposed strong bounds on the speed of gravitational waves (GWs) locally, inferring that the speed of GWs propagation is equal to the speed of light. Current GW detectors in operation will not be able to observe BNS merger to long cosmological distance, where possible cosmological corrections on the cosmic expansion history are expected to play an important role, specially for investigating possible deviations from general relativity. Future GW detectors designer projects will be able to detect many coalescences of BNS at high $z$, such as the third generation of the ground GW detector called Einstein Telescope (ET) and the space-based detector deci-hertz interferometer gravitational wave observatory (DECIGO). In this paper, we relax the condition $c_{T} / c=1$ to investigate modified GW propagation where the speed of GWs propagation is not necessarily equal to the speed of light. Also, we consider the possibility for the running of the Planck mass corrections on modified GW propagation. We parametrize both corrections in terms of an effective GW luminosity distance and we perform a forecast analysis using standard siren events from BNS mergers, within the sensitivity predicted for the ET and DECIGO. We find at high $z$ very strong forecast bounds on the running of the Planck mass, namely $\mathcal{O}\left(10^{-1}\right)$ and $\mathcal{O}\left(10^{-2}\right)$ from ET and DECIGO, respectively. Possible anomalies on GW propagation are bound to $\left|c_{T} / c-1\right| \leq 10^{-2}\left(10^{-2}\right)$ from ET (DECIGO), respectively. We finally discuss the consequences of our results on modified gravity phenomenology.
\end{abstract}

Keywords: Modified Gravity, Gravitational Waves Standard Sirens 


\section{Contents}

1 Introduction 1

2 Gravitational Waves in Modified Gravity 2

3 Methodology and Results 5

4 Implications on modified gravity phenomenology 14

5 Final remarks $\quad 15$

A Checking the impact of the galaxy velocity dispersion $\quad 15$

\section{Introduction}

Astronomical information from gravitational wave (GW) observations will open a new and wide spectrum of possibilities to investigate fundamental physics, which might shed light to clarify open questions in modern cosmology, especially regarding the dark sector of the Universe. One of the most fascinating and important sources of GWs is certainly the gravitational radiation emitted from binary neutron star (BNS) mergers. At present, one BNS merger event has been detected, the GW170817 event [1], accompanied by its electromagnetic counterpart, the GRB $170817 \mathrm{~A}$ event [2], located at $40 \mathrm{Mpc}(z \approx 0.01)$. This event was also the first standard siren (SS) observation, the GWs analog of astronomical standard candles, and opened the window for the multi-messenger GW astronomy (we refer the reader to [3] for a summary of all GW detections up to the present time). Although the GW170817 event is located at very low $z$, preliminary cosmological information and consequences of this observation are important to the understanding of our Universe locally. These observations were also used to measure $H_{0}$ at $12 \%$ accuracy [4]. An improvement of this result was presented in $[5,6]$, while we refer to [7-9] for proposals to use SS to measure $H_{0}$ with more accuracy in the near future. A very important consequence of this BNS signals was the strong bound placed on the GW speed, $\left|c_{T} / c-1\right| \leq 10^{-16}$, where $c_{T}$ and $c$ are the propagation speed of the GWs and light, respectively. In practical physical terms, this means that, locally, the speed of GWs propagation is equal to the speed of light. Strong constraints have been also imposed on modified gravity/dark energy scenarios [10-13]. See also [14-19] for discussion of the speed of GWs propagation on modified gravity models.

The detectability rate of the BNS merger with their electromagnetic counterpart from the current LIGO/VIRGO sensitivity is expected to be low for the next years. Also, the sensitivity of these detectors will not able to detect BNS merger at high $z$. The central importance of GW astronomy is testified by the plans for construction of several GW observatory interferometers, beyond the present performance of the LIGO and Virgo interferometers, such as Cosmic Explore [20], Einstein Telescope (ET) [21, 22], LISA [23], DECIGO [24], TianQin [25], IMAGEM [26], among others, to observe GWs in the most diverse frequencies bands and different types of GW sources. Certainly, the most promising projects to detect SS events from BNS merger will be the ET and DECIGO detectors, given their frequency bands and 
sensitivity designs. These detectors will be able to detect thousands of GWs events with great accuracy, and have been widely used for cosmological constraint investigations [27-47].

In this work, setting $c_{T} / c=1$ for $z<0.1$ (cosmological distance ten times greater than GW170817), we relax the condition $c_{T} / c=1$ at high $z$ and we perform a forecast analysis on the ratio $c_{T} / c$, up to $z=2$, from some modified gravity parametric models where the speed of GWs propagation is not necessarily equal to the speed of light. Also, we consider the possibility for the running of the Planck mass corrections on modified GW propagation. To this purpose, we generated standard siren mock catalogs from BNS mergers, within the sensitivity predicted for the ET and DECIGO.

The paper is structured as follows. In Section 2, we present the theoretical scenario in which the GW propagation is modified when $c_{T} / c \neq 1$, as well as also in presence of the running of the Planck mass corrections. In Section 3, we describe briefly our methodology and the main forecast analysis results. In Section 4, we discuss consequences of our results on modified gravity models. Lastly, in Section 5 we outline our final considerations and perspectives. Throughout the text, the prime symbols indicate derivatives with respect to the conformal time, and a subscript zero refers to a quantity evaluated at the present time.

\section{Gravitational Waves in Modified Gravity}

The most general tensor metric perturbation evolution, under the FLRW metric, can be written as [48]

$$
h_{A}^{\prime \prime}+(2+\nu) \mathcal{H} h_{A}^{\prime}+\left(c_{T}^{2} k^{2}+\mu^{2}\right) h_{A}=\Pi_{A},
$$

where $h_{A}$ is the metric tensor perturbation, being $A=\{+, \times\}$ the label of the two polarization states, and $\mathcal{H}$ is the Hubble rate in conformal time. The quantities $\nu, c_{T}$ and $\mu$ represent the running of the effective Planck mass, the GW propagation speed and the effective graviton mass, respectively. The function $\Pi_{A}$ denotes extra sources generating GWs, which we assume to be null. The running of the Planck mass enters as a friction term and it is responsible for modifying the amplitude of the tensor modes, acting as a damping term during the cosmic time. This is also related to the strength of gravity. The term $c_{T}^{2} k^{2}+\mu^{2}$ accounts for modifications of the GW phase. In general, all these functions depend on the parameters of a specific theory (e.g., see $[49,50]$ and references therein for more details on the physical interpretation of these functions).

Recently, it has been discussed that modifications in the underlying gravity theory can affect not only the generation mechanism (waveform of the GWs), but also their propagation through cosmological distances [51, 52]. Since the GW amplitude is inversely proportional to the luminosity distance, the modification in the amplitude and phase coming from Eq. (2.1) can be interpreted as a correction to the GW luminosity distance on general theories of modified gravity (see also $[49,53]$ ). Assuming theories with $\mu=0$ and for tensor modes inside the horizon, where the GW wavelength is much smaller than the cosmological horizon, we have that the effective GW luminosity distance for non-trivial function $\nu$ and $c_{T}$ satisfies the equation ${ }^{1}$

$$
d_{L}^{G W}(z)=\sqrt{\frac{c_{T}(z)}{c_{T}(0)}} \exp \left[\frac{1}{2} \int_{0}^{z} \frac{d z^{\prime}}{1+z^{\prime}} \nu\left(z^{\prime}\right)\right] \times(1+z) \int_{0}^{z} \frac{c_{T}\left(z^{\prime}\right) d z^{\prime}}{H\left(z^{\prime}\right)},
$$

\footnotetext{
${ }^{1}$ For a didactic deduction of Eq. (2.2), see Appendix A in [54].
} 
where, for $\nu=0$ and $c_{T}=1$, we recover the general relativity case ( $\Lambda \mathrm{CDM}$ cosmology), that is, $d_{L}^{G W}(z)=d_{L}^{E M}(z)$, where $d_{L}^{E M}$ is the standard luminosity distance for an electromagnetic signal. Generalizations and interpretations of some effective GW luminosity distance have been recently studied in the context of modified gravity theories (see, e.g., [55-67]).

In order to move on, we need to specify a gravitational model. A common procedure is to choose phenomenologically functional forms to model the dynamics of $\nu$ and $c_{T}$. In the recent literature, this has been done through $\alpha_{i}$ 's functions (see, e.g., [68-70]), especially to investigate the Horndeski gravity and beyond. The quantities $\nu$ and $c_{T}$ are well modeled for $\alpha_{M}$ (Planck-mass run rate) and $\alpha_{T}$ (tensor speed excess), respectively, following the relationships $\nu=\alpha_{M}$ and $c_{T}^{2}(z)=1+\alpha_{T}(z)$. Typically, the $\alpha_{i}$ 's evolution is given as some power $n$ of the scale factor $a(t)$ or the dark energy density $\Omega_{d e}(a)$. In the present work, we adopt two parametrizations for $\alpha_{i}$ :

I. First, let us consider the well-known form $\alpha_{i}=\alpha_{i 0} a^{n}$, where the label $i$ refers to $M$ and $T$. Denoting by $n_{1}$ and $n_{2}$ the powers over the Planck-mass run rate and tensor speed excess, respectively, we thus have $\alpha_{M}=\alpha_{M 0} a^{n_{1}}$ and $\alpha_{T}=\alpha_{T 0} a^{n_{2}}$. We will refer to this scenario as model $I$.

II. In order to check for possible dependence on the parametrization, we propose a new $\alpha_{T}$ parametrization, which is characterized by a smoother parametric dynamics with respect to the previous model:

$$
\alpha_{T}=\alpha_{T 0} \tanh \left(1-a^{\tau}\right),
$$

where $\tau>0$ is a constant. Our parametrization, which will be called model II in what follows, presents some advantages in comparison with model I. The $\tau$ parameter controls the shape of the cosmic evolution of $c_{T}(z)$, determining the possible amount of variation from the speed of light at high $z$. For positive values of $\tau, c_{T}$ asymptotically tends to $c$ at low $z$, and the higher the value of $\tau$, the faster $c_{T} \rightarrow c$ at small cosmological distances, while $c_{T} / c \neq 1$ at high $z$ in such a way to be constant for $z \gg 1$. In Figure 1, we quantify these effects for reasonable values of $\alpha_{T 0}$ and $\tau$. In this scenario, we keep $\alpha_{M}=\alpha_{M 0} a^{n_{1}}$ for the running of the Planck mass.

An important role is played by the stability conditions of the theory. Suitable values of the free parameters must be considered to have a stable theory throughout the evolution of the Universe (see [70] and reference therein). The stability conditions for $\alpha_{M}=\alpha_{M 0} a^{n_{1}}$ can be summarized as follows:

$$
\begin{aligned}
& n_{1}>\frac{5}{2}, \alpha_{M 0}<0 \\
& 0<n_{1}<1+\frac{3 \Omega_{m 0}}{2}, \alpha_{M 0}>0
\end{aligned}
$$

where $\Omega_{m 0}$ is the present normalized matter density. Moreover, the stability of the tensor modes requires $c_{T}^{2}(z)=1+\alpha_{T}(z)>0$. In the following, we assume these conditions in our results for both models.

In Figure 2, we show the corrections due to model I on the effective GW luminosity distance induced from both contributions $\alpha_{T 0}$ and $\alpha_{M 0}$, inducing $d_{L}^{G W}>d_{L}^{E M}$ and $d_{L}^{G W}<$ $d_{L}^{E M}$ for $\alpha_{M 0}>0$ and $\alpha_{M 0}<0$, respectively. In drawing the plot, we keep $\alpha_{M 0}$ fixed and 


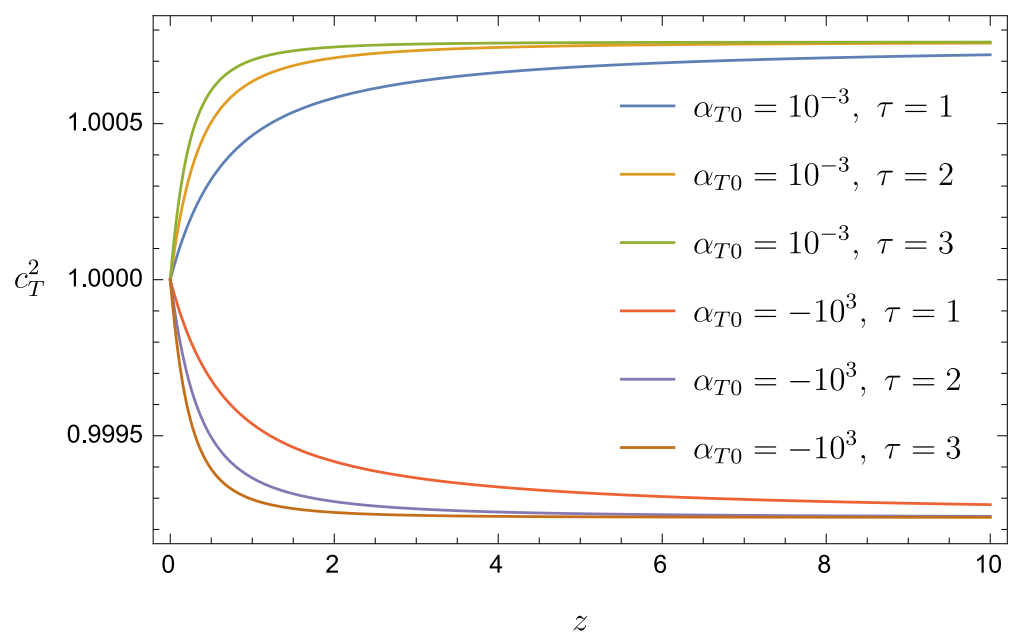

Figure 1: GW propagation speed as a function of the redshift for the tensor speed excess parametrization defined in 2.3, for different values of the free parameters of the theory.
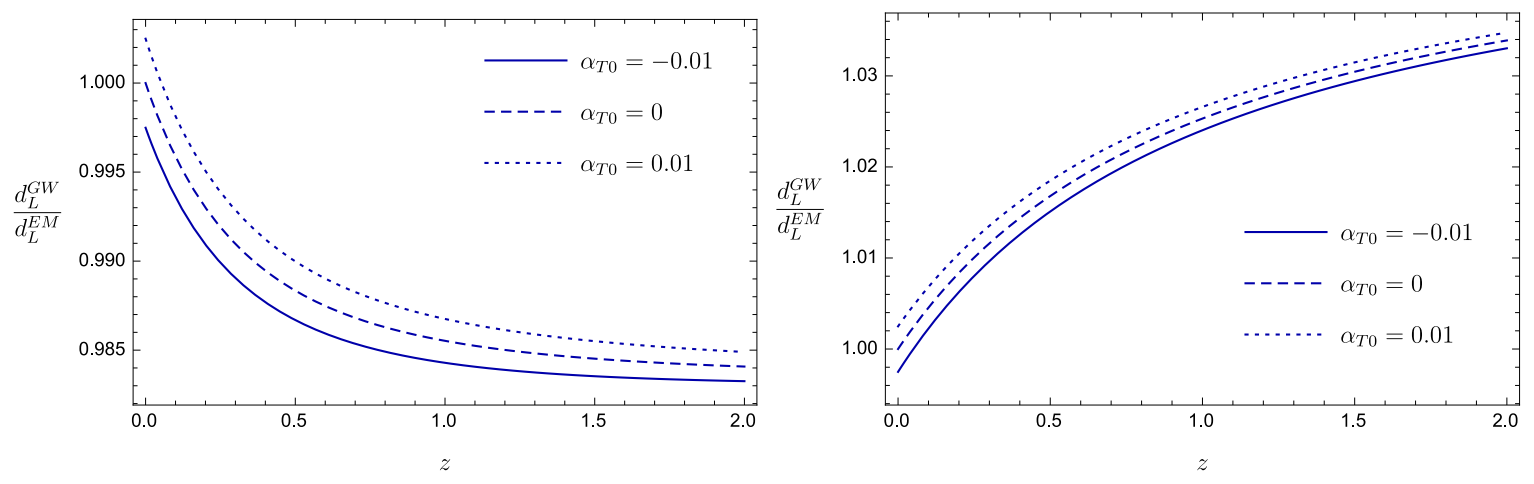

Figure 2: Corrections on the effective GW luminosity distance (cf. Eq. (2.2)) as a function of the redshift for model $I$ with different values of the tensor speed excess $\alpha_{T 0}$ with fixed values of $\alpha_{M 0}$. Left panel: $\alpha_{M 0}=-0.1, n_{1}=3, n_{2}=1$. Right panel: $\alpha_{M 0}=0.1, n_{1}=1$, $n_{2}=1$. The limit $d_{L}^{E M}(z) / d_{L}^{E M}=1$ represents general relativity.

we vary $\alpha_{T 0}$ between $[-0.01,0.01]$ for both stability conditions $\alpha_{M 0}>0,<0$. We note that for $\alpha_{M 0}<0$, corrections with positive (negative) values of $\alpha_{T 0}$ will induce $d_{L}^{G W}>d_{L}^{E M}$ $\left(d_{L}^{G W}<d_{L}^{E M}\right)$, compared to the $\alpha_{T 0}=0$ prevision. While, when considering $\alpha_{M 0}>0$, for $\alpha_{T 0}>0(<0)$ we have $d_{L}^{G W}>d_{L}^{E M}\left(d_{L}^{G W}<d_{L}^{E M}\right)$. Therefore, the determination of the signal, imposed by the stability conditions, provides a variety of possible corrections on the effective luminosity distance.

In Figure 3, we show the corrections on $d_{L}^{G W} / d_{L}^{E M}$ predicted by model II. We soon notice different properties in our proposal. In all the cases, considering reasonable values of all free parameters of the theory ( $\alpha_{T}$ and $\alpha_{M}$ functions), we find the convergence $d_{L}^{G W} / d_{L}^{E M} \rightarrow 1$ at low $z$ and corrections $d_{L}^{G W} \neq d_{L}^{E M}$ at high $z$. The presence of a possible time variation of the Planck-mass will determine $d_{L}^{G W}<d_{L}^{E M}$ (when $\alpha_{M 0}<0$ ) or $d_{L}^{G W}>d_{L}^{E M}$ (when $\alpha_{M 0}>0$ ). The sign determination of $\alpha_{T 0}$ will mitigate possible corrections $d_{L}^{G W} \neq d_{L}^{E M}$ at high $z$ (see 

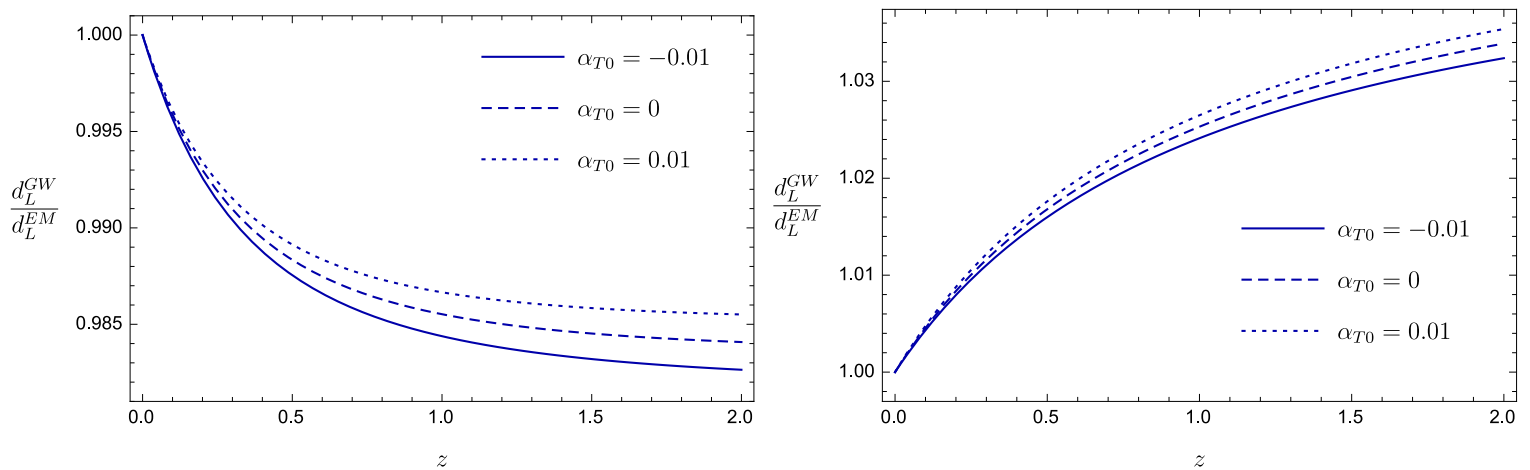

Figure 3: Corrections on the effective GW luminosity distance taking into account the new parametrization (2.3), i.e. model II, for different values of the tensor speed excess $\alpha_{T 0}$ with fixed values of $\alpha_{M 0}$. Left panel: $\alpha_{M 0}=-0.1, n_{1}=3, \tau=1$. Right panel: $\alpha_{M 0}=0.1$, $n_{1}=1, \tau=1$.

Figure 3).

In the following, we will forecast bounds on the free parameters for all the scenarios using mock catalogs of the standard siren events from BNS mergers.

\section{Methodology and Results}

We shall now analyze the waveform emitted by the binary system. For a given a GW strain signal $h(t)=A(t) \cos [\Phi(t)]$, one can use the stationary-phase approximation for the orbital phase of inspiraling binary system to obtain its Fourier transform $\tilde{h}(f)$. In the case of a coalescing binary system of masses $m_{1}$ and $m_{2}$, we have

$$
\tilde{h}(f)=Q \mathcal{A} f^{-7 / 6} e^{i \Phi(f)},
$$

where $\mathcal{A} \propto 1 / d_{L}^{G W}$ is the modified amplitude given by Eq. (2.2). The $\Phi(f)$ is the inspiral phase of the binary system. More details on the post-Newtonian coefficients and waveforms can be found in [59] and references therein.

Once the modified GW signal propagation is defined, for a high enough signal-to-noise ratio (SNR), we can obtain upper bounds on the free parameters of the GW signal $\tilde{h}(f)$ by means of the Fisher information analysis. Estimating $d_{L}(z)$ from GW standard sirens mock data is a well consolidated methodology, and we refer to [59] for a detailed description. Pioneer studies in this regard are represented by the works $[71,72]$. In what follows, we briefly describe our methodology.

We considered the ET and DECIGO power spectral density noises to generate our mock standard siren catalogs. Figure 4 shows the spectral noise density curve of both experiments. ET is a third-generation ground detector, covering the frequency range $1-10^{4} \mathrm{~Hz}$. The signal amplitude which ET is sensitive to is expected to be ten times larger than the current advanced ground-based detectors. The ET conceptual design study predicts an order of $10^{3}-10^{7}$ BNS detections per year. Nevertheless, only a small fraction $\left(\sim 10^{-3}\right)$ of them is expected to be accompanied by a short $\gamma$-ray burst observation. Assuming a detection rate of $\mathcal{O}\left(10^{5}\right)$, the events with short $\gamma$-ray bursts will be $\mathcal{O}\left(10^{2}\right)$ per year. DECIGO is the most sensitive GW detector proposed in the $0.1-10 \mathrm{~Hz}$ band, enough to detect a cosmological 


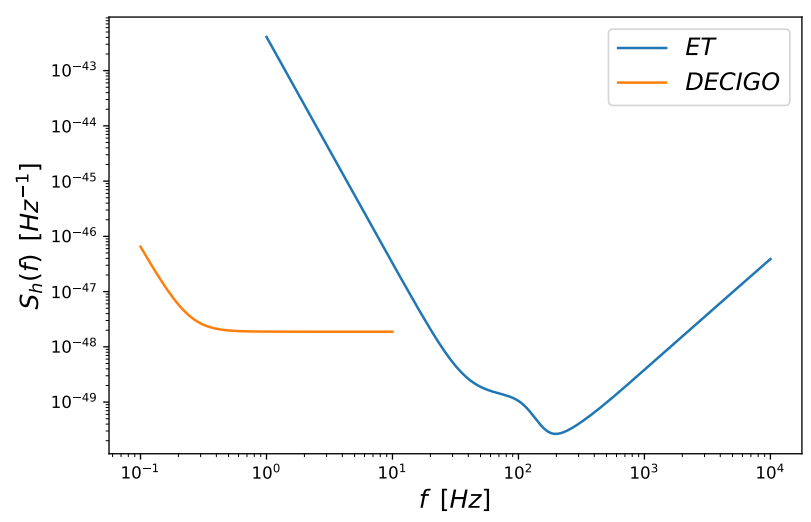

Figure 4: Detector spectral noise density for the Einstein Telescope (ET) and deci-hertz interferometer gravitational wave observatory (DECIGO).

GW background generated at early times, the mergers of intermediate-mass black holes and a large number of BNS merges. These GW sources enable us to measure the cosmological expansion with unprecedented precision [40-45] and to test alternative theories of gravity [73-75]. Based on this setup, in [44] it has been shown that cosmological parameters can be accurately measured by DECIGO with a precision of $\sim 1 \%$ assuming a large number of BNS.

In our simulations, we considered 1000 BNS mock GW standard sirens merger events, up to $z=2$, for the ET and DECIGO detectors. The redshift distribution of the sources, taking into account evolution and stellar synthesis, is well described by

$$
P(z) \propto \frac{4 \pi \chi^{2}(z) R(z)}{H(z)(1+z)}
$$

where $\chi$ is the comoving distance and $R(z)$ describes the time evolution of the burst rate and is given by $R(z)=1+2 z$ for $z<1, R(z)=3 / 4(5-z)$ for $1 \leq z \leq 5$, and $R(z)=0$ for $z>5$. The input masses of the NSs in our mock catalogs are randomly sampled from uniform distributions within $[1-2] \mathrm{M}_{\odot}$. When generating our mock GW events, we have only considered mergers with SNR $>8^{2}$.

The redshift range and event numbers rate are fully compatible with the sensitivity of both instruments. For each event, in each catalogue, we estimate the measurement error on the luminosity distance for the ET and DECIGO configurations by applying the Fisher matrix analysis on the waveforms (see [59] for details). We then construct the Fisher matrix for the parameters of the cosmological model under consideration as

\footnotetext{
${ }^{2} \mathrm{~A}$ relevant quantity for generating the mock catalogue is the signal-to-noise ratio (SNR) associated with each simulated event. The SNR is given by:

$$
\mathrm{SNR}^{2} \equiv 4 R e \int_{f_{\min }}^{f_{\max }} d f \frac{|h(f)|^{2}}{S_{n}},
$$

where $S_{n}(f)$ is the spectral noise density. The upper cutoff frequency $f_{\max }$ is determined by the last stable orbit. The lower cutoff frequency $f_{\min }$ is instead determined for each specific GWs detector.
} 


$$
F_{i j}=\sum_{n} \frac{1}{\sigma_{\text {ins }}^{2}+\sigma_{\text {lens }}^{2}\left(z_{n}\right)+\sigma_{v}^{2}\left(z_{n}\right)} \frac{\partial d_{L}\left(z_{n}\right)}{\partial \theta_{i}} \frac{\partial d_{L}\left(z_{n}\right)}{\partial \theta_{j}},
$$

where the sum $n$ runs over all standard sirens mock events. The derivatives are performed with respect to the cosmological parameters $\theta_{i}=\left\{H_{0}, \Omega_{m 0}, \alpha_{M 0}, \alpha_{T 0}, n_{1}, n_{2}(\tau)\right\}$ evaluated at their fiducial input values. In our analysis, we used $\theta_{i}=\{67.4,0.30,0.0,0.0,3.0(1.0), 1.0(1.0)\}$ as fiducial values. The $n_{1}$ values have been chosen to fulfil the stability conditions (2.4)-(2.5) from $\alpha_{M 0}<0(>0)$. The quantities $\sigma_{\text {ins }}^{2}, \sigma_{\text {lens }}^{2}\left(z_{n}\right)$ and $\sigma_{v}^{2}\left(z_{n}\right)$ are the instrumental, lensing and galaxy peculiar velocity errors, respectively. For both instruments, we consider the galaxy peculiar velocity error given by [76]

$$
\sigma_{v}^{2}=d_{L} \times\left|1-\frac{(1+z)^{2}}{H(z) d_{L}}\right| \sigma_{\mathrm{v}, \text { gal }}
$$

where $\sigma_{\mathrm{v}, \text { gal }}$ is the one-dimensional velocity dispersion of the galaxy, set to be $300 \mathrm{~km} / \mathrm{s}$, as a rough estimate. It is well known that systematic effects due $\sigma_{v}^{2}$ are relevant only at very low $z$. In Appendix A, we show how much different $\sigma_{\mathrm{v} \text {,gal }}$ values can affect our simulations. As we are interested in simulated data at high $z$, the effects produced by $\sigma_{\mathrm{v} \text {,gal }}$ are minimal in our forecast analysis. Thus, without loss of generality, let us assume $300 \mathrm{~km} / \mathrm{s}$ in all the forecast analyses performed in this work.

For ET, the other uncertainties can be summarized as [28]

$$
\sigma_{\mathrm{ins}}^{2}+\sigma_{\text {lens }}^{2}=\left(\frac{2 d_{L}}{\mathrm{SNR}}\right)^{2}+\left(0.05 z d_{L}\right)^{2},
$$

while, for DECIGO, we have [77]

$$
\sigma_{\text {ins }}^{2}+\sigma_{\text {lens }}^{2}=\left(\frac{2 d_{L}}{\mathrm{SNR}}\right)^{2}+d_{L} \times 0.066\left[\frac{1-(1+z)^{-0.25}}{0.25}\right]^{1.8} .
$$

Thus, the total uncertainty on the measurement for each experiment is given by

$$
\sigma_{d_{L}}=\sqrt{\sigma_{\text {ins }}^{2}+\sigma_{\text {lens }}^{2}+\sigma_{v}^{2}} .
$$

We calculated the SNR of each event and confirmed that it is a GW detection if SNR > 8. When performing the integration, we assumed $f_{\text {low }}=1(0.1) \mathrm{Hz}$ for ET (DECIGO). As for the upper frequency limit, we considered $f_{\text {upper }}=2 f_{L S O}$ for both cases, where $f_{L S O}=1 /\left(6^{3 / 2} 2 \pi M_{z}\right)$ is the orbital frequency at the last stable orbit, with $M_{z}=(1+z) M$. In the DECIGO case, if $f_{\text {upper }}>10 \mathrm{~Hz}$ for any event, then we fixed $f_{\text {upper }}=10 \mathrm{~Hz}$. More details about NSs parameters distributions can be found in [59].

Table 1 and 2 summarize the forecast constraints at the $95 \%$ confidence level (C.L.) on the parameters of the theory for models I and II, respectively. Figures 5 and 6 shows the full parameter space from both stability conditions on the running of the effective Planck mass, where a direct comparison on each parameter from each experiment can be seen for model I. Similarly, in Figures 7 and 8, we show the parameter space concerning model II.

We are particularly interested in the limits on $\alpha_{M 0}$ and $\alpha_{T 0}$, especially on $\alpha_{T 0}$, to quantify deviations on the speed of GW propagation. Thus, let us discuss in detail our results on these parameters. For all scenarios, we note that the observational bonds from 


\begin{tabular}{c|cc|cc}
\hline \hline & & Stability condition $\alpha_{M 0}<0$ & Stability condition $\alpha_{M 0}>0$ & \\
\hline Parameter & $\sigma(\mathrm{ET})$ & $\sigma(\mathrm{DECIGO})$ & $\sigma(\mathrm{ET})$ & $\sigma($ DECIGO $)$ \\
\hline$\alpha_{T 0}$ & 0.053 & 0.015 & 0.057 & 0.016 \\
$\alpha_{M 0}$ & $>-0.18$ & $>0.050$ & $<0.087$ & $<0.052$ \\
$n_{1}$ & 0.41 & 0.13 & 0.33 & 0.12 \\
$n_{2}$ & 0.18 & 0.053 & 0.17 & 0.052 \\
\hline \hline
\end{tabular}

Table 1: Forecast constraints from the ET and DECIGO experiments for model $I$ under both stability conditions $\alpha_{M 0}<0,>0$. The notations $\sigma(\mathrm{ET})$ and $\sigma$ (DECIGO) represent the 95\% C.L. uncertainties on the fiducial input values.

\begin{tabular}{|c|c|c|c|c|}
\hline & & ty condition $\alpha_{M 0}<0$ & Stability condition $\alpha_{M 0}>0$ & \\
\hline Parameter & $\sigma(\mathrm{ET})$ & $\sigma(\mathrm{DECIGO})$ & $\sigma(\mathrm{ET})$ & $\sigma(\mathrm{DECIGO})$ \\
\hline$\alpha_{T 0}$ & 0.11 & 0.036 & 0.11 & 0.036 \\
\hline$\alpha_{M 0}$ & $>-0.16$ & $>-0.050$ & $<0.079$ & $<0.023$ \\
\hline$n_{1}$ & 0.41 & 0.13 & 0.34 & 0.11 \\
\hline$\tau$ & 0.21 & 0.047 & 0.21 & 0.048 \\
\hline
\end{tabular}

Table 2: Forecast constraints from the ET and DECIGO experiments for model II under both the stability conditions $\alpha_{M 0}<0,>0$. The notation is the same as in Table 1 .

DECIGO sensitivity are significantly improved when compared to ET. In fact, this is expected as DECIGO is more sensitive in amplitude than ET (cf. Figure 4) at low frequencies, where the binary system is still emitting signals, thus making possible to detect BNS mergers events with higher SNR values with respect to ET. Therefore, the errors measurement of the free parameters of the theory will be more accurate in the DECIGO experiment due to the higher SNR associated with the events in this band. This can be quantified analyzing the Figure of Merit (FoM) for the estimated sensitivities of the parameters $\alpha_{T 0}$ and $\alpha_{M 0}$ on its corresponding plane as

$$
\operatorname{FoM}(M)=|F|^{1 / 2} \frac{\Gamma(M / 2+1)}{\pi^{M / 2}}\left(\delta \chi^{2}\right)^{-M / 2},
$$

where

$$
\delta \chi^{2}(M, n)=2, \quad \mathcal{G}\left[\frac{M}{2}, 1-\operatorname{erf}\left(\frac{n}{\sqrt{2}}\right)\right],
$$

being $\mathcal{G}$ the inverse of the $\Gamma$ regularized function. Here, $M$ is the number of parameters of the theory, erf is the error function, $n$ is the level of statistical confidence desired, and $|F|$ is the determinant of Fisher Matrix $F_{i j}$ [78]. The above definition offers the advantage to make useful comparisons for different dark energy experiments or, in this case, for future GW experiments with respect to their sensitivity in constraining different cosmological parameters. Higher values of the FoM correspond to tighter constraints on model I and model II. For model I, specifically in the case of $\alpha_{M 0}<0$, we find that the sensitivities on $\left(\alpha_{T 0}, \alpha_{M 0}\right)$ from the DECIGO experiment $(\mathrm{FoM}=40.941)$ improves the FoM from the ET experiment $(\mathrm{FoM}=3.498)$ by a factor of $\sim 11.704$, while, in the case with $\alpha_{M 0}>0$, we find $\mathrm{FoM}=27.221$ and FoM $=5.163$ from the DECIGO and ET experiments respectively, with an improvement by a factor of $\sim 5.272$ (see Figures 6 and 5). For model II, we found the 


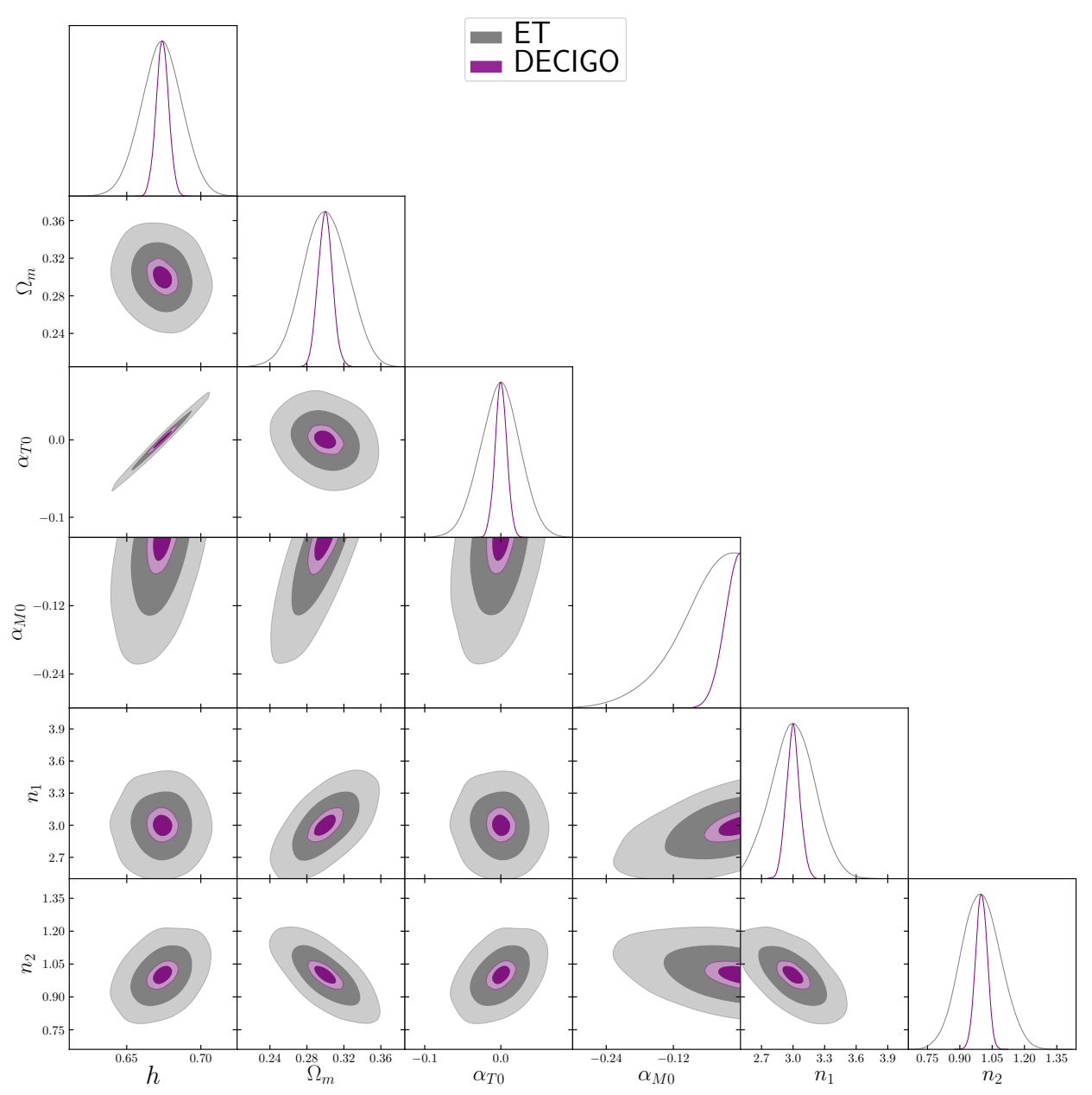

Figure 5: One-dimensional marginalized distribution and $68 \%$ and $95 \%$ C.L. forecasted regions for the parameters of model $I$ from the ET and DECIGO experiments. The stability condition $\alpha_{M 0}<0$ is considered.

following results for the same set of parametric spaces: for $\alpha_{M 0}<0$, DECIGO experiment $(\mathrm{FoM}=22.052)$ improves the FoM from the ET experiment $(\mathrm{FoM}=2.683)$ by a factor of $\sim 8.219$ and for the case with $\alpha_{M 0}>0$, we found FoM $=33.166$ and FoM $=3.932$ from the DECIGO and ET experiments respectively, with an improvement of $\sim 8.434$ (Figures 8 and 7). This represents a great performance on the whole baseline of the scenarios under consideration.

Our forecast analysis strongly bounds possible deviations from general relativity. This is evident from the forecast bounds on the running of the Planck mass, where for the model I, we find: $-0.18(-0.05)<\alpha_{M 0} \leq 0$ from ET (DECIGO) and $0 \leq \alpha_{M 0}<0.087(0.052)$ from ET (DECIGO), under the conditions $\alpha_{M 0}<0$ and $\alpha_{M 0}>0$, respectively. In the case of model II, we find: $-0.16(-0.05)<\alpha_{M 0} \leq 0$ from ET (DECIGO) and $0 \leq \alpha_{M 0}<$ 0.079 (0.023) from ET (DECIGO), respectively. These limits appear significantly more robust than the current constraints [79-81], being our results able to foresee strongly bounds on some phenomenological gravity models. 


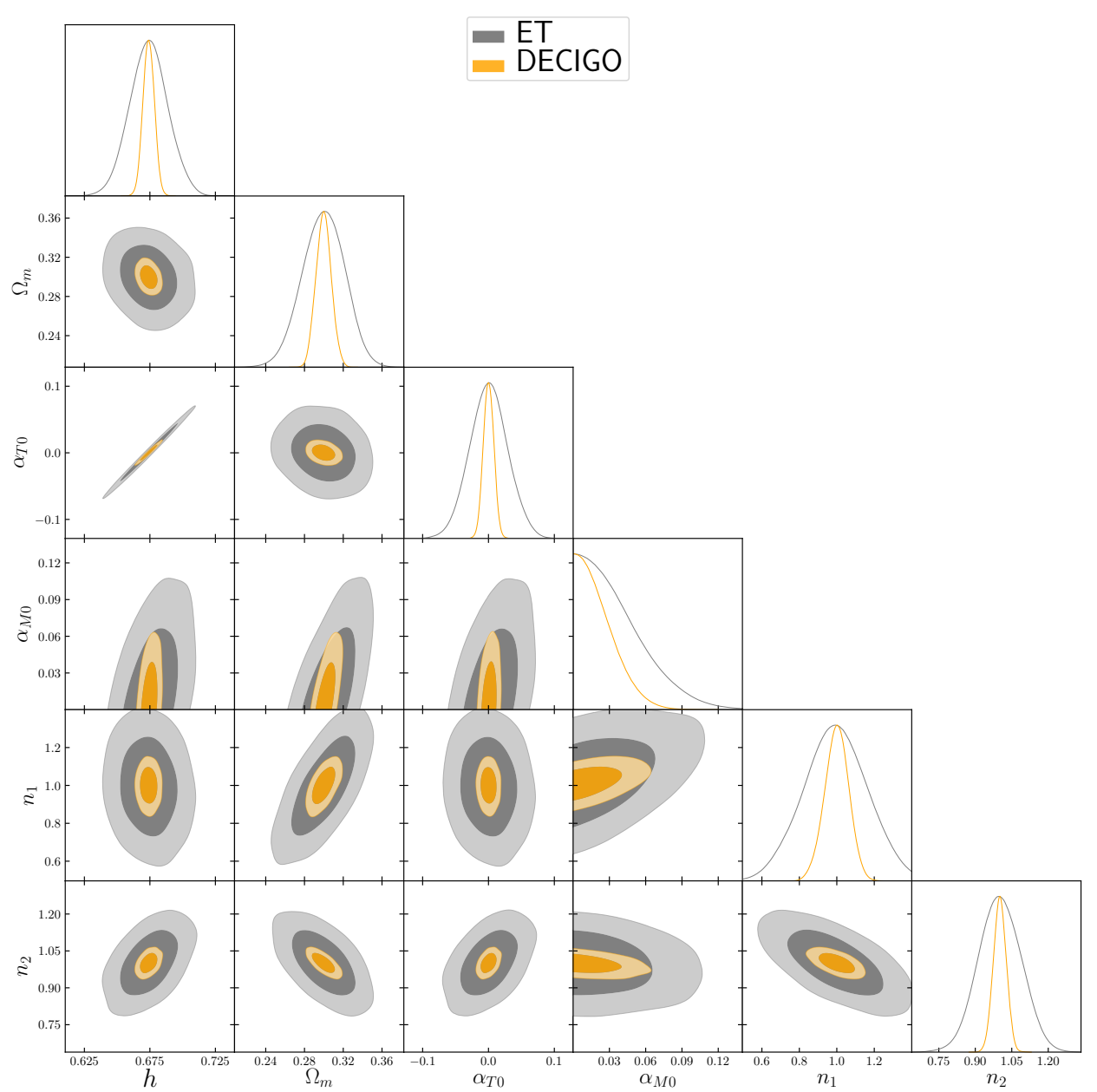

Figure 6: One-dimensional marginalized distribution and $68 \%$ and $95 \%$ C.L. forecasted regions for the parameters of model I from the ET and DECIGO experiments. The stability condition $\alpha_{M 0}>0$ is considered.

The main aim of this work is to find new limits on $\alpha_{T 0}$ and, as expected, we obtained $\left|\alpha_{T 0}\right| \ll 1$. In particular, we find that $\alpha_{T 0}$ can be tightly constrained when analyzed by DECIGO, with an improvement of one order of magnitude with respect to ET. It is important to emphasize that, to the authors' knowledge, the forecast analysis on $\alpha_{T 0}$ has never been performed in the literature from the point of view of standard sirens GWs data. Therefore, we shall discuss two different perspectives in this context, from two different future GW experiments.

For model I with $\alpha_{M 0}<0$, we find $\alpha_{T 0} \sim \mathcal{O}\left(10^{-2}\right), \mathcal{O}\left(10^{-2}\right)$ from ET and DECIGO, respectively. When considering $\alpha_{M 0}>0$, we note that the forecast error on $\alpha_{T 0}$ are similar to when assuming $\alpha_{M 0}<0$. This shows that the forecast sensitivity on $\alpha_{T 0}$ within this parametrization is minimally dependent on the stability conditions induced from the Planckmass run rate.

Assuming our proposal, i.e. model II, we also find that the forecast on $\alpha_{T 0}$ minimally depends on the sign of $\alpha_{M 0}$. On the other hand, we note a difference on the tensor speed 


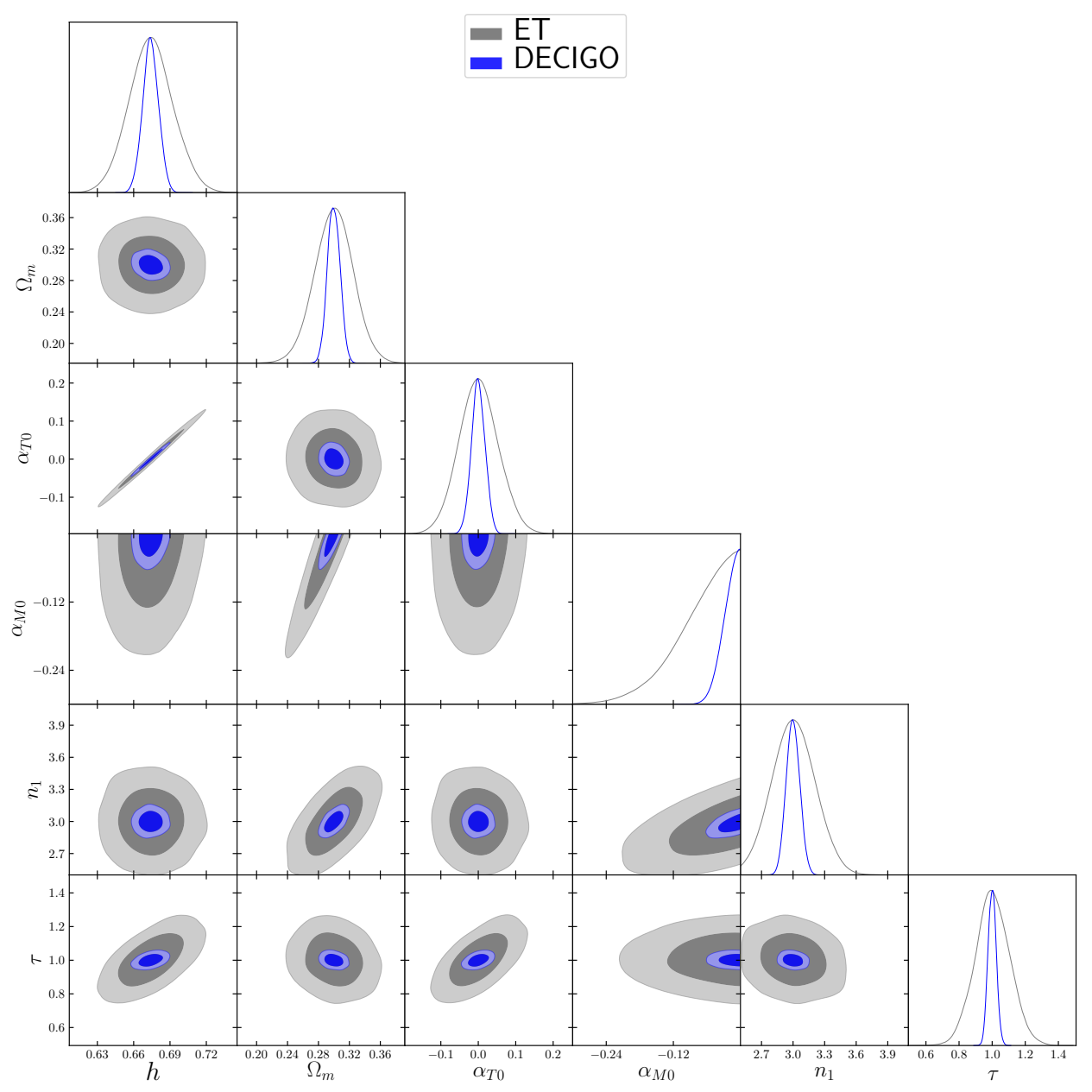

Figure 7: One-dimensional marginalized distribution and $68 \%$ and $95 \%$ C.L. forecasted regions for the parameters of model II from the ET and DECIGO experiments. The stability condition $\alpha_{M 0}<0$ is considered.

excess with forecast sensitivity given by $\alpha_{T 0} \sim \mathcal{O}\left(10^{-1}\right), \mathcal{O}\left(10^{-2}\right)$ from ET and DECIGO, respectively: the DECIGO sensitivity can improve up to one order of magnitude in comparison to ET. Apparently, different parametrizations can reasonably change the simulations (the forecast analysis) on the $\alpha_{T 0}$ parameter. Due to this possible bias dependence in the theoretical input, we shall argue in the following why our phenomenological proposal is significantly more appropriate to describe the evolution of $c_{T}(z)$.

It is interesting to compare our simulations with results obtained by other authors. In [82], combining future missions of stage IV photometric redshift and CMB surveys, the authors found $\alpha_{T 0} \sim \mathcal{O}\left(10^{-1}\right)$. Thus, a direct comparison between these and our estimates reveals that forthcoming GWs data have the potential to constrain $\alpha_{T 0}$ with an accuracy up to one orders of magnitude greater when compared to future CMB and LSS surveys. Proposals to measure the speed of GWs in the pre-recombination era are presented in [83].

Let us now look at measurements from already catalogued data. In [84] combinations of various data, including Planck data, showed that from CMB is possible to obtain $\alpha_{T 0} \sim$ 


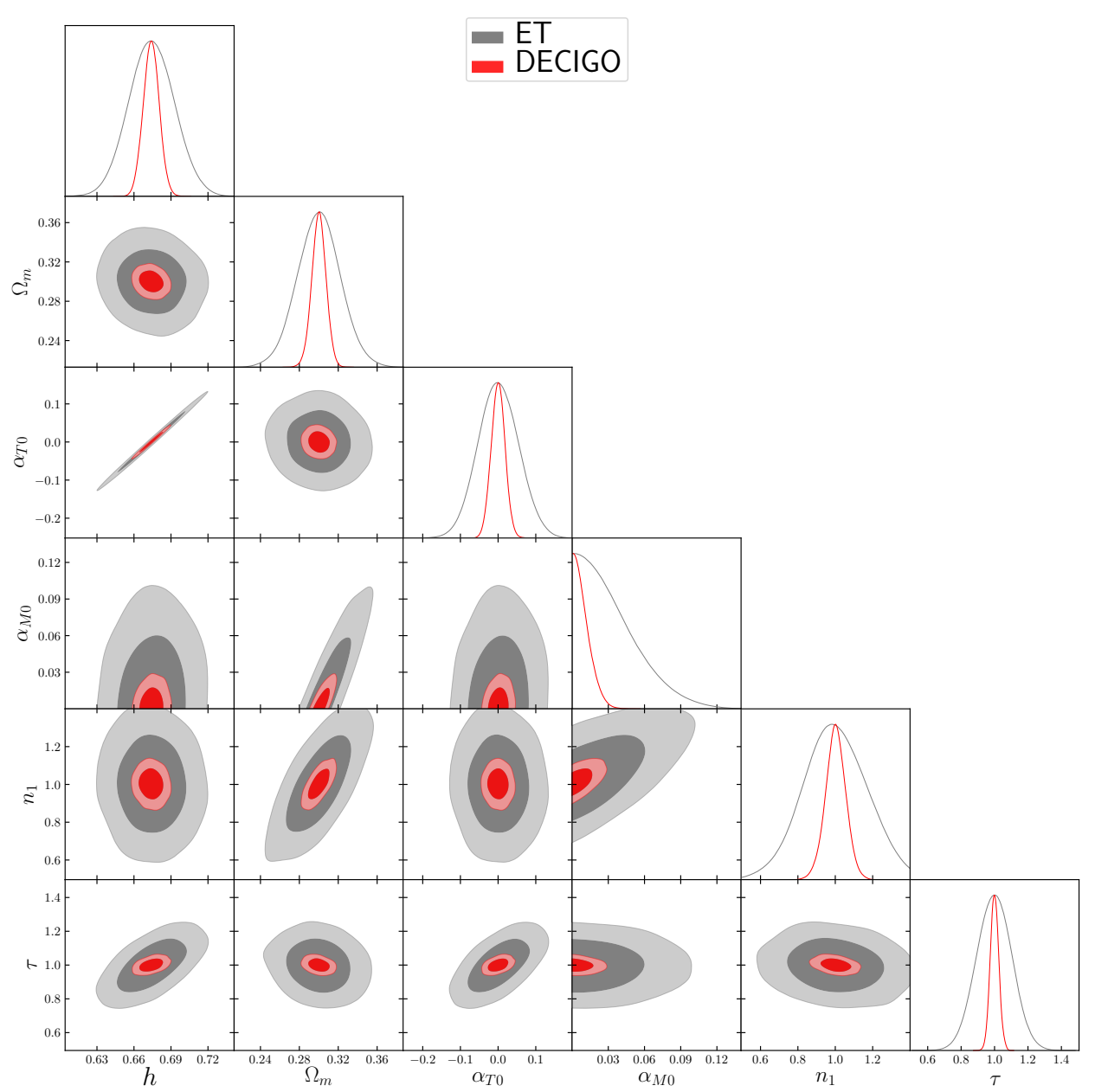

Figure 8: One-dimensional marginalized distribution and $68 \%$ and $95 \%$ C.L. forecasted regions for the parameters of model II from the ET and DECIGO experiments. The stability condition $\alpha_{M 0}>0$ is considered.

$\mathcal{O}\left(10^{-1}\right)$, and with significant preference for $\alpha_{T 0}<0$ from a robust joint analysis with multiple data (see table 4 there). In [85], the authors find $0.55<c_{T} / c<1.42$ from the time delay between GWs signals arriving at widely separated detectors.

Therefore, standard sirens merger events from the ET perspectives allow to get bounds on $\alpha_{T 0}$ compatible with the limits obtained via current and future CMB and LSS data, and these bounds can be improved up to one order of magnitude using the DECIGO design. On the other hand, it is important to note that all of these bounds on possible anomalies in GWs propagation quantified at high $z$ are several orders of magnitude larger than those provided by the GW170817 event locally, where in fact no corrections on general relativity should be expected. Hence, investigating $c_{T}(z)$ at very high $z$ can open new perspectives in this sense, to test gravity models where in principle $c_{T} / c \neq 1$.

In Figure 9, we show the reconstruction of $c_{T}^{2}$ for model I, in units of $c=1$, as a function of the cosmic time up to $z=2$ (statistical limit information of our mock SS catalogs). In the reconstruction, we considered $z=0.5$ as a cut-off, in order to quantify $c_{T}^{2}$ at high $z$ only. We 

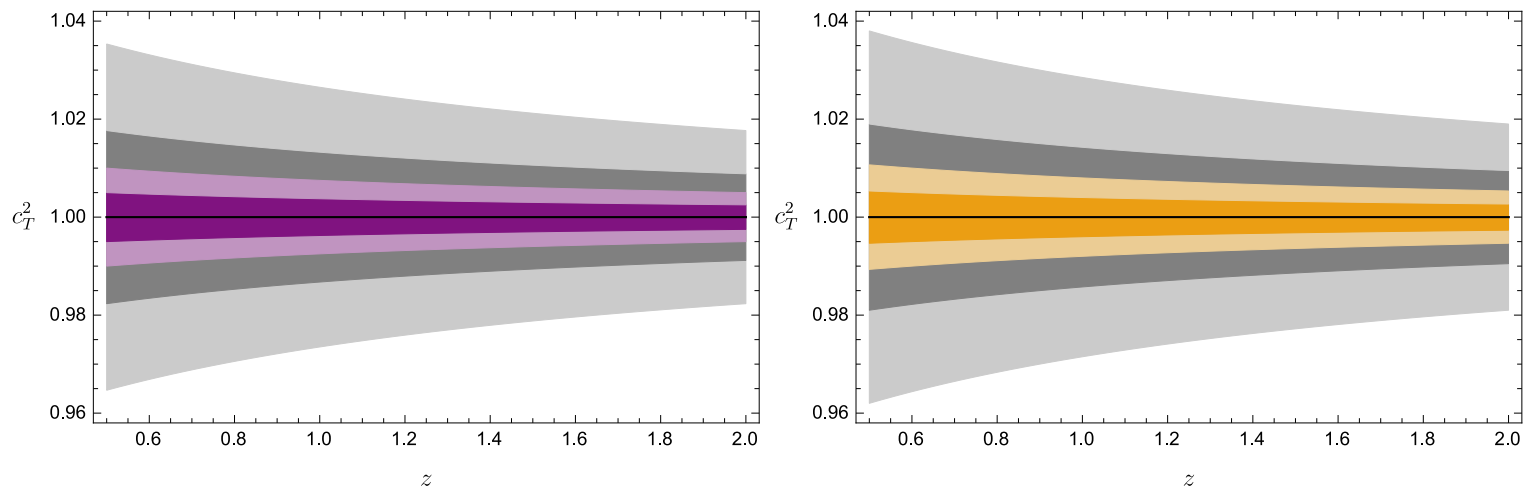

Figure 9: Reconstruction of $c_{T}^{2}$ for $\alpha_{M 0}<0$ (left panel) and $\alpha_{M 0}>0$ (right panel) using the $68 \%$ and $95 \%$ C.L. results for model $I$ from the Fisher information analysis on the ET and DECIGO experiments. The colours match those of Figures 5 and 6.
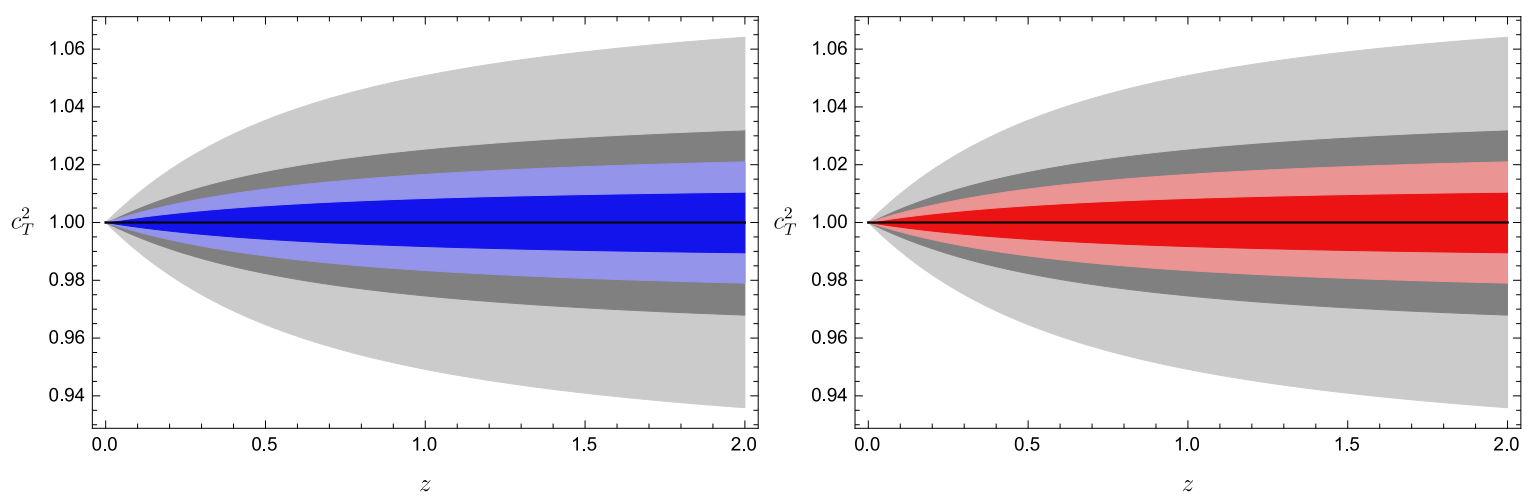

Figure 10: Same as in Figure 9, but for model II. The colours match those of Figures 7 and 8.

remind that, in our analysis, we assume $c_{T} / c=1$ at low $z$, motivated by GW170917 event. Thus, we are bounding $c_{T}^{2}$ at high $z$ in such a way to be compatible with GW170917 at very low $z$. The reconstruction is done for both experiments and, as expected, the constraints are stronger in the case of DECIGO. The statistical bounds are insensitive to the stability condition on $\alpha_{M 0}$. We find that $c_{T}^{2}$ can deviate from general relativity $3.5 \%(1.8 \%)$ at the $68 \%$ $(95 \%)$ C.L. from ET and $1 \%(0.5 \%)$ at the $68 \%$ (95\%) C.L. from DECIGO, with maximum deviations at intermediate $z$. These deviations tend to become smaller at larger $z$.

In Figure 10, we show the reconstruction up to the 95\% C.L. of $c_{T}^{2}$ for model II. To analyze the main differences between the two different parametrizations, we show the reconstruction of $c_{T}(z)$ for model II over the whole $z$ range considered in the GWs experiments. In particular, we can see that $c_{T} \rightarrow 1$ for low $z$, where possible deviations from $c_{T} / c \neq 1$ are strongly bound and disadvantaged from both detectors sensitivities. This characteristic is not present in the parametrization commonly taken into account in the literature (model I analyzed here), where possible anomalies on the speed of GWs propagation show up at low $z$ (it can be seen intuitively in Figure 9). Possible corrections, $c_{T} / c \neq 1$, in model II 
are manifested only at intermediate and high $z$, with a maximum deviation from general relativity of $2 \%(6 \%)$ at the $68 \%(95 \%)$ C.L. from ET and $1 \%(0.5 \%)$ at the $68 \%$ (95\%) C.L. from DECIGO, at very high $z$. In this model, corrections of the same order of magnitude hold up to the CMB scale $(z \sim 1100)$. Thus, as a conservative estimate, we can conclude that possible anomalies on the speed of GWs under model II are about $[0-2] \%$ and $[0-$ $0.5] \%$ at the $68 \%$ C.L. from the ET and DECIGO sensitivities, respectively, from very low to very high $z$.

The dynamic feature inherent of our proposal is interesting in direct comparison with the model I. While in model II we can obtain possible deviations to be provided in the CMB scale until the strong bond imposed by the GW170917 event at very low- $z$, the same dynamics on model I is only possible with a very rigid $z$ cut-off at intermediate $z$. Thus, analyzing only its dynamic character, we can claim that our proposal is more realistic and compatible with observations.

\section{Implications on modified gravity phenomenology}

We here briefly discuss the implications of our forecast analysis on some examples of modified gravity models. Although our bounds on $\alpha_{T 0}$ and $\alpha_{M 0}$ have been obtained parametrically, without loss of generality, we can follow the opposite path and consider appropriate models in order to study consequences on some scenarios. Since model II provides a better dynamics in view of a direct comparison of the $c_{T}(z)$ cosmic evolution with different observations, we will take the results from this case only in our discussions in this section. However, similar conclusions can be easily drawn from the results obtained using model I. Within the Horndeski theories of gravity [86-88], the GWs propagation speed can be described by $c_{T}^{2}=1+\alpha_{T}[68]$, and one has

$$
M_{*}^{2} \alpha_{T}=2 X\left(2 G_{4 X}-2 G_{5 \phi}-(\ddot{\phi}-\dot{\phi}) G_{5 X}\right),
$$

where the functions $G_{4,5}$ depend on the scalar field $\phi$ and $X \equiv-1 / 2\left(\nabla^{\mu} \phi \nabla_{\mu} \phi\right)$ is the kinetic term. The function $M_{*}^{2}$ is the rate of evolution of the effective Planck mass.

As a first example, we consider the derivative coupling theory in which the scalar field couples to the Einstein tensor in the form $\xi \phi G_{\mu \nu} \nabla^{\mu} \nabla^{\nu} \phi[89,90]$. The parameter $\xi$ represents the coupling constant of the theory and quantifies possible anomalies on the GWs speed propagation. The general relativity case is recovered when $\xi=0$. In the Horndeski Lagrangian formalism, this scenario corresponds to $G_{4}=M_{p l}^{2} / 2$ and $G_{5}=\xi \phi$. Then, we can write the tensor speed excess as $M_{*}^{2} \alpha_{T}=2 \xi \dot{\phi}^{2}$. Thus, the speed of GWs is given by

$$
c_{T}^{2}=1+\frac{2 \dot{\phi}^{2}}{M_{*}^{2}} \xi .
$$

At intermediate $z$, it is reasonable to assume $\dot{\phi} / M_{*} \simeq 1$, so that we estimate $-0.005 \lesssim$ $\xi \lesssim 0.005(-0.0018 \lesssim \xi \lesssim 0.0018)$ at the $95 \%$ C.L. from ET (DECIGO), in the case $\alpha_{M 0}<0$. Similar conclusions, in terms of order of magnitude on $\xi$, are obtained when considering the stability condition $\alpha_{M 0}>0$.

A second well-known scenario where $c_{T} \neq c$ are the covariant Galileons models [91, 92], which are characterized by the functions $G_{4}=M_{p l}^{2} / 2+\beta_{4} X^{2}$ and $G_{5}=\beta_{5} X^{2}$, where $\beta_{4}$ and $\beta_{5}$ are constants. This generates a non-trivial evolution for the speed of GWs, namely

$$
c_{T}^{2}=1+\frac{\dot{\phi}^{4}}{M_{*}^{2}}\left[2 \beta_{4}-\beta_{5}(\ddot{\phi}-\dot{\phi})\right] .
$$


In the particular cases $\beta_{4}=\beta_{5}=0$ and $\beta_{5}=0$, we obtain the cubic and quartic Galileon scenarios, respectively. For a qualitative discussion, we consider the quartic Galileon model, for which we find the constraint $-0.005 \lesssim 2 \beta_{4} \dot{\phi}^{4} / M_{*}^{2} \lesssim 0.005$ at the $95 \%$ C.L from ET sensitivity. An exact and accurate solution for $\phi(t)$ would require a thorough investigation of the cosmic dynamics, but assuming that the field does not vary much with respect to Planck mass, within the validity range of our analysis, we can estimate $\left|\beta_{4}\right| \sim \mathcal{O}\left(10^{-3}\right)$. In general, the full dynamics of the model should be consistent with the aforementioned range.

Many other gravity models predict possible anomalies on GWs propagation, where in general our parametric boundaries can be used to impose some limitation on such theories. It is not our main aim to make an exhaustive qualitative comparison with the most diverse scenarios. In short, our results show that for any and all models that theoretically predict $c_{T} / c \neq 1$, this ratio must be approximately in the range $0.97 \lesssim c_{T} / c \lesssim 1.03$ and $0.99 \lesssim$ $c_{T} / c \lesssim 1.01$ at the $68 \%$ C.L. as predicted by ET and DECIGO, respectively, at very high $z$ scale, for both stability conditions on the running of the Planck mass, when analyzed within the perspective of model II.

\section{Final remarks}

Modifications of the general relativity theory are motivated mainly to explain the dark sector of the Universe. Due to extra degrees of freedom of gravitational origin, modified gravity models predict physical properties beyond the standard features of general relativity. Among several consequences, many theories call for possible anomalies on GWs propagation. The observation of the GW170817 event has imposed that the speed of GWs is equal to the speed of light for scales less than $40 \mathrm{Mpc}$. This puts strong limits and discards any model that predicts $c_{T} \neq c$ within this cosmological scale. However, the dark energy effects are manifested on large scales. Motivated by this aspect, we thus performed a forecast analysis using 1000 standard siren events from BNS mergers, within the sensitivity predicted for ET and DECIGO up to $z=2(\sim 15539 \mathrm{Mpc})$. In so doing, we searched for a new bound on the ratio $c_{T} / c$ when considering modifications on GWs propagation between source and detector, considering corrections on the speed of GWs. We found $\left|c_{T} / c-1\right| \leq 10^{-2}\left(10^{-2}\right)$ from ET (DECIGO) assuming model I, and $\left|c_{T} / c-1\right| \leq 10^{-1}\left(10^{-2}\right)$ from ET (DECIGO) for model II, which leaves room for small possible corrections predicted by alternative theories, compared to the only information from GW170817 event at very low $z$. Our analysis relies on a simple functional form of $\alpha_{T}$, and other more robust parametric functions may be used to model the $c_{T}(z)$ dynamics more effectively. Nevertheless, the main findings of this work represent the first forecast analysis obtained by using information from SS mock data from future detector design. In this respect, our results open a new window for possible tests on $c_{T}(z)$ in the future.

\section{A Checking the impact of the galaxy velocity dispersion}

In this Appendix, we study the impact of different galaxy velocity dispersions on our forecast analysis. As known, the galaxy peculiar velocity error has only significant influence on verylow $z$ events. Figure 11 quantifies this point by showing the ratio between the galaxy peculiar velocity error on the total error estimative on $d_{L}(z)$ as a function of $z$. We note that at high $z$, where we are particularly interested, the effects due to $\sigma_{\mathrm{v} \text {,gal }}$ should not significantly influence 


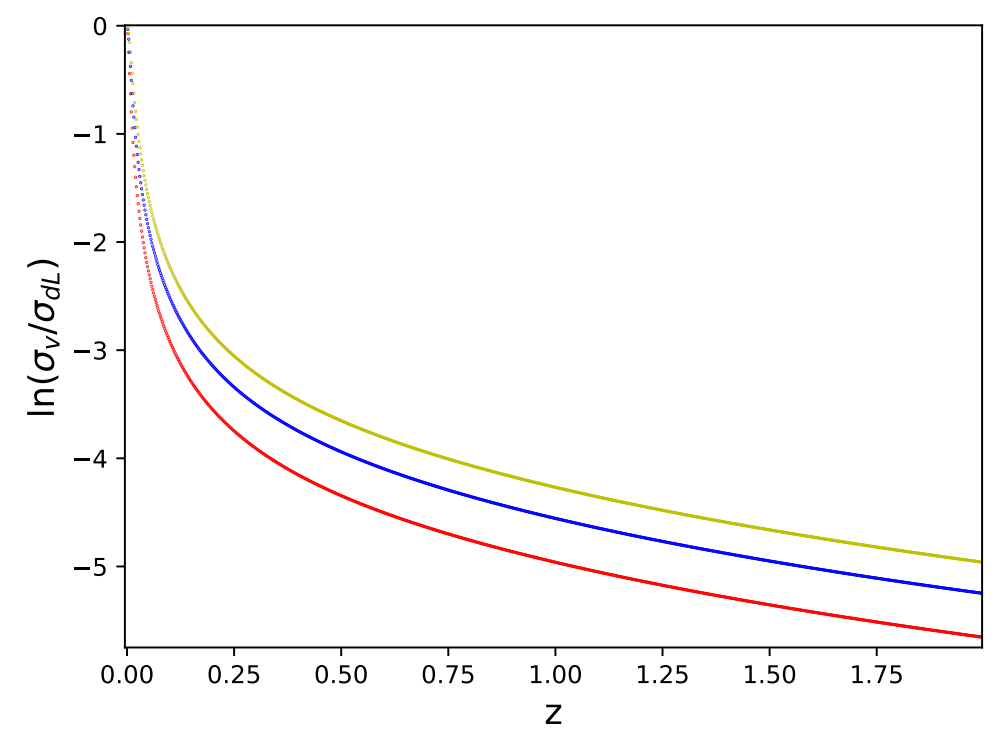

Figure 11: Ratio between the galaxy peculiar velocity error $\left(\sigma_{\mathrm{v}, \text { gal }}\right)$ and the total error $\left(\sigma_{\mathrm{d}_{\mathrm{L}}}\right)$ as a function of the redshift for different galaxy velocity dispersion values on a logarithmic scale: $200 \mathrm{~km} / \mathrm{s}$ (red), $300 \mathrm{~km} / \mathrm{s}$ (blue) and $400 \mathrm{~km} / \mathrm{s}$ (yellow).

the forecast analysis on large cosmological distances. In fact, $\sigma_{\mathrm{v}, \text { gal }}$ only contributes on scales corresponding to $z \ll 1$, with particular significant effects at $z<0.15$.

In Tables 3, 4, and 5, we summarize our results for model I considering different galaxy velocity dispersions: $200 \mathrm{~km} / \mathrm{s}, 300 \mathrm{~km} / \mathrm{s}$ and $400 \mathrm{~km} / \mathrm{s}$. As expected, we did not notice significant changes in our main results when considering different $\sigma_{\mathrm{v}, \text { gal }}$ within the range [200 - 400$] \mathrm{km} / \mathrm{s}$, being all bounds compatible with each other. Furthermore, since we considered the $\Lambda \mathrm{CDM}$ model previsions for $z<0.10$ in order to be locally compatible with the GW170817 event at very low $z$, in the region where we have any possible effects from $\sigma_{\mathrm{v}}$, gal, i.e. for $z \ll 1$, we have the same dynamics as predicted by general relativity. Without loss of generality, the same arguments can be applied when considering the model II.

\begin{tabular}{c|cc|cc}
\hline \hline & & Stability condition $\alpha_{M 0}<0$ & Stability condition $\alpha_{M 0}>0$ & \\
\hline Parameter & $\sigma(\mathrm{ET})$ & $\sigma($ DECIGO $)$ & $\sigma(\mathrm{ET})$ & $\sigma($ DECIGO $)$ \\
\hline \hline$\alpha_{T 0}$ & 0.054 & 0.016 & 0.056 & 0.018 \\
$\alpha_{M 0}$ & $>-0.18$ & $>-0.054$ & $<0.089$ & $<0.030$ \\
$n_{1}$ & 0.40 & 0.13 & 0.33 & 0.11 \\
$n_{2}$ & 0.23 & 0.061 & 0.22 & 0.060 \\
\hline \hline
\end{tabular}

Table 3: Forecast constraints from the ET and DECIGO experiments, assuming velocity dispersion of the galaxy set to be $200 \mathrm{~km} / \mathrm{s}$ and under both stability condition $\alpha_{M 0}<0,>0$ for all free parameter of the theory. The notation is the same as in Table 1. 


\begin{tabular}{c|cc|cc}
\hline \hline & & Stability condition $\alpha_{M 0}<0$ & Stability condition $\alpha_{M 0}>0$ & \\
\hline Parameter & $\sigma(\mathrm{ET})$ & $\sigma($ DECIGO $)$ & $\sigma(\mathrm{ET})$ & $\sigma($ DECIGO $)$ \\
\hline$\alpha_{T 0}$ & 0.053 & 0.015 & 0.057 & 0.016 \\
$\alpha_{M 0}$ & $>-0.18$ & $>-0.050$ & $<0.087$ & $<0.052$ \\
$n_{1}$ & 0.41 & 0.13 & 0.33 & 0.12 \\
$n_{2}$ & 0.18 & 0.053 & 0.17 & 0.052 \\
\hline \hline
\end{tabular}

Table 4: Same as in Table 3, but assuming velocity dispersion of the galaxy set to be 300 $\mathrm{km} / \mathrm{s}$.

\begin{tabular}{c|cc|cc}
\hline \hline & \multicolumn{3}{|c}{ Stability condition $\alpha_{M 0}<0$} & Stability condition $\alpha_{M 0}>0$ \\
\hline Parameter & $\sigma(\mathrm{ET})$ & $\sigma(\mathrm{DECIGO})$ & $\sigma(\mathrm{ET})$ & $\sigma(\mathrm{DECIGO})$ \\
\hline$\alpha_{T 0}$ & 0.055 & 0.014 & 0.060 & 0.016 \\
$\alpha_{M 0}$ & $>-0.19$ & $>-0.050$ & $<0.090$ & $<0.024$ \\
$n_{1}$ & 0.45 & 0.13 & 0.34 & 0.11 \\
$n_{2}$ & 0.22 & 0.051 & 0.21 & 0.049 \\
\hline \hline
\end{tabular}

Table 5: Same as in Table 3, but assuming velocity dispersion of the galaxy set to be 400 $\mathrm{km} / \mathrm{s}$.

\section{Acknowledgments}

The authors thank the referee for his/her valuable comments and suggestions. RD acknowledges the support of INFN (iniziativa specifica QGSKY). RCN would like to thank the Brazilian agency FAPESP for financial support under Project No. 2018/18036-5. JCNA would like to thank FAPESP for financial support under Project No. 2013/26258-4, and CNPq for partial financial support under Grant No. 307217/2016-7.

\section{References}

[1] B. P. Abbott et al., GW170817: Observation of Gravitational Waves from a Binary Neutron Star Inspiral, Phys. Rev. Lett. 119 (2017) 161101 [arXiv:1710.05832].

[2] B. P. Abbott et al., Gravitational Waves and Gamma-Rays from a Binary Neutron Star Merger: GW170817 and GRB 170817A, Astrophys. J. 848 (2017) L13 [arXiv:1710.05834].

[3] B. P. Abbott et al., GWTC-1: A Gravitational-Wave Transient Catalog of Compact Binary Mergers Observed by LIGO and Virgo during the First and Second Observing Runs, Phys. Rev. X 9 (2019) 031040 [arXiv:1811.12907].

[4] B. P. Abbott et al., A gravitational-wave standard siren measurement of the Hubble constant, Nature 551 (2017) 7678 [arXiv:1710.05835].

[5] K. Hotokezaka et al., A Hubble constant measurement from superluminal motion of the jet in GW170817, Nature Astron. 3 (2019) 940 [arXiv:1806.10596].

[6] M. S. Santos et al., First Measurement of the Hubble Constant from a Dark Standard Siren using the Dark Energy Survey Galaxies and the LIGO/Virgo BinaryBlack-hole Merger GW170814, Astrophys. J. 876 (2019) L7 [arXiv:1901.01540].

[7] H. Y. Chen, M. Fishbach and D. E. Holz, A two per cent Hubble constant measurement from standard sirens within five years, Nature 562 (2018) 7728 [arXiv:1712.06531]. 
[8] S. M. Feeney et al., Prospects for Resolving the Hubble Constant Tension with Standard Sirens, Phys. Rev. Lett. 122 (2019) 061105 [arXiv:1802.03404].

[9] A. Nishizawa, Measurement of Hubble constant with stellar-mass binary black holes, Phys. Rev. D 96 (2017) 101303 [arXiv:1612.06060].

[10] T. Baker et al., Strong Constraints on Cosmological Gravity from GW170817 and GRB 170817A, Phys. Rev. Lett. 119 (2017) 251301 [arXiv:1710.06394].

[11] P. Creminelli and F. Vernizzi, Dark Energy after GW170817 and GRB170817A, Phys. Rev. Lett. 119 (2017) 251302 [arXiv:1710.05877].

[12] J. Sakstein and J. Jain, Implications of the Neutron Star Merger GW170817 for Cosmological Scalar-Tensor Theories, Phys. Rev. Lett. 119 (2017) 251303 [arXiv:1710.05893].

[13] J. M. Ezquiaga and M. Zumalacrregui, Dark Energy After GW170817: Dead Ends and the Road Ahead, Phys. Rev. Lett. 119 (2017) 251304 [arXiv:1710.05901].

[14] C. Dalang and L. Lombriser, Limitations on Standard Sirens tests of gravity from screening, JCAP 10 (2019) 013 [arXiv:1906.12333].

[15] J. B. Jimenez, F. Piazza and H. Velten, Evading the Vainshtein Mechanism with Anomalous Gravitational Wave Speed: Constraints on Modified Gravity from Binary Pulsars, Phys. Rev. Lett. 116 (2016) 061101 [arXiv:1507.05047].

[16] D. Bettoni, J. M. Ezquiaga, K. Hinterbichler and M. Zumalacarregui, Speed of gravitational waves and the fate of scalar-tensor gravity, Phys. Rev. D 95 (2017) 084029 [arXiv:1608.01982].

[17] A. Nishizawa and T. Nakamura, Measuring Speed of Gravitational Waves by Observations of Photons and Neutrinos from Compact Binary Mergers and Supernovae, Phys. Rev. D 90, 044048 (2014) arXiv:1406.5544 [gr-qc].

[18] A. Nishizawa, Constraining the propagation speed of gravitational waves with compact binaries at cosmological distances, Phys. Rev. D 93, 124036 (2016) arXiv:1601.01072 [gr-qc].

[19] X. Gao and X. Y. Hong, Propagation of the gravitational waves in a cosmological background, arXiv:1906.07131 [gr-qc].

[20] L. S. Collaboration, Instrument science white paper, Ligo-t1400316-v4.

[21] Einstein Telescope Science Team, ET design study document, ET-0106C-10.

[22] M. Punturo et al., The third generation of gravitational wave observatories and their science reach, Class. Quant. Grav. 27 (2010) 173001, Proceedings of the 8th Edoardo Amaldi Conference on Gravitational Waves (Amaldi 8), Columbia University, New York, June 2009.

[23] H. Audleyet et al., Laser Interferometer Space Antenna (2017) [arXiv:1702.00786].

[24] N. Seto, S. Kawamura and T. Nakamura, Possibility of Direct Measurement of the Acceleration of the Universe Using $0.1 \mathrm{~Hz}$ Band Laser Interferometer Gravitational Wave Antenna in Space, Phys. Rev. Lett. 87 (2001) 221103 [astro-ph/0108011].

[25] J. Luo et al., TianQin: a space-borne gravitational wave detector, Class. Quant. Grav. 33 (2016) 035010 [arXiv:1512.02076].

[26] M. Tinto, R. C. Nunes, M. E. S. Alves, J. C. N. de Araujo et al., In Preparation.

[27] B. S. Sathyaprakash, B. F. Schutz and C. Van Den Broeck, Cosmography with the Einstein Telescope, Class. Quant. Grav. 27 (2010) 215006 [arXiv:0906.4151].

[28] W. Zhao, C. Van Den Broeck, D. Baskaran and T. G. F. Li, Determination of dark energy by the Einstein Telescope: Comparing with CMB, BAO, and SNIa observations, Phys. Rev. D 83 (2011) 023005 [arXiv:1009.0206]. 
[29] R. G. Cai and T. Yang, Estimating cosmological parameters by the simulated data of gravitational waves from the Einstein Telescope, Phys. Rev. D 95 (2017) 044024 [arXiv:1608.08008].

[30] M. Du, W. Yang, L. Xu, S. Pan and D. F. Mota, Future constraints on dynamical dark-energy using gravitational-wave standard sirens, Phys. Rev. D 100 (2019) 043535 [arXiv:1812.01440].

[31] W. Yang, S. Pan, E. Di Valentino, B. Wang and A. Wang, Forecasting Interacting Vacuum-Energy Models using Gravitational Waves [arXiv:1904.11980].

[32] J. J. Wei, Model-independent Curvature Determination from Gravitational-wave Standard Sirens and Cosmic Chronometers, Astrophys. J. 868 (2018) 29 [arXiv:1806.09781].

[33] X. N. Zhang, L. F. Wang, J. F. Zhang and X. Zhang, Improving cosmological parameter estimation with the future gravitational-wave standard siren observation from the Einstein Telescope, Phys. Rev. D 99 (2019) 063510 [arXiv:1804.08379].

[34] L. F. Wang, X. N. Zhang, J. F. Zhang and X. Zhang, Impacts of gravitational-wave standard siren observation of the Einstein Telescope on weighing neutrinos in cosmology, Phys. Lett. B 782 (2018) 87 [arXiv:1802.04720].

[35] J. F. Zhang, H. Y. Dong, J. Z. Qi and X. Zhang, Prospect for constraining holographic dark energy with gravitational wave standard sirens from the Einstein Telescope [arXiv:1906.07504].

[36] J. Z. Qi et al., Testing the Etherington distance duality relation at higher redshifts: Combined radio quasar and gravitational wave data, Phys. Rev. D 99, 063507 (2019), arXiv:1902.01988 [astro-ph.CO].

[37] W. Yang et al., Listening to the sound of dark sector interactions with gravitational wave standard sirens, JCAP 1907 (2019) 037 [arXiv:1905.08286].

[38] X. Fu, L. Zhou and J. Chen, Testing the cosmic distance-duality relation from future gravitational wave standard sirens, Phys. Rev. D 99 (2019) 083523 [arXiv:1903.09913].

[39] W. Yang, S. Pan, D. F. Mota and M. Du, Forecasting Dark-Energy Anisotropic Stress using gravitational-waves [arXiv:2001.02180].

[40] C. Cutler and D. E. Holz, Ultrahigh precision cosmology from gravitational waves, Phys. Rev. D 80 (2009) 104009 [arXiv:0906.3752].

[41] A. Nishizawa, A. Taruya and S. Kawamura, Cosmological test of gravity with polarizations of stochastic gravitational waves around $0.11 \mathrm{~Hz}$, Phys. Rev. D 81 (2010) 104043 [arXiv:0911.0525].

[42] A. Nishizawa, A. Taruya and S. Saito, Tracing the redshift evolution of Hubble parameter with gravitational-wave standard sirens, Phys. Rev. D 83 (2011) 084045 [arXiv:1011.5000].

[43] K. Yagi, Scientific Potential of DECIGO Pathfinder and Testing GR with Space-Borne Gravitational Wave Interferometers, Int. J. Mod. Phys. D 22 (2013) 1341013 [arXiv:1302.2388].

[44] R. C. Nunes, M. E. S. Alves and J. C. N. de Araujo, Primordial gravitational waves in Horndeski gravity, Phys. Rev. D 99 (2019) 084022 [arXiv:1811.12760].

[45] R. G. Cai, T. B. Liu, X. W. Liu, S. J. Wang and T. Yang, Probing cosmic anisotropy with gravitational waves as standard sirens, Phys. Rev. D 97 (2018) 103005 [arXiv:1712.00952].

[46] Z. Carson, B. C. Seymour and K. Yagi, Future Prospects for Probing Scalar-Tensor Theories with Gravitational Waves from Mixed Binaries arXiv:1907.03897 [gr-qc].

[47] Z. Carson and K. Yagi, Parameterized and inspiral-merger-ringdown consistency tests of gravity with multi-band gravitational wave observations arXiv:1911.05258 [gr-qc].

[48] I. D. Saltas, I. Sawicki, L. Amendola and M. Kunz, Anisotropic Stress as a Signature of Nonstandard Propagation of Gravitational Waves, Phys. Rev. Lett. 113 (2014) 191101 [arXiv:1406.7139]. 
[49] A. Nishizawa, Generalized framework for testing gravity with gravitational-wave propagation. I. Formulation, Phys. Rev. D 97 (2018) 104037 [arXiv:1710.04825].

[50] J. M. Ezquiaga and M. Zumalacrregui, Dark Energy in Light of Multi-Messenger Gravitational-Wave Astronomy, Front. Astron. Space Sci. 5 (2018) 44 [arXiv:1807.09241].

[51] E. Belgacem, Y. Dirian, S. Foffa, and M. Maggiore, Gravitational-wave luminosity distance in modified gravity theories, Phys. Rev. D 97 (2018) 104066 [arXiv:1712.08108].

[52] E. Belgacem, Y. Dirian, S. Foffa, and M. Maggiore, Modified gravitational-wave propagation and standard sirens, Phys. Rev. D 98 (2018) 023510 [arXiv:1805.08731].

[53] S. Arai and A. Nishizawa, Generalized framework for testing gravity with gravitational-wave propagation. II. Constraints on Horndeski theory, Phys. Rev. D 97 (2018) 104038 [arXiv:1711.03776].

[54] E. Belgacem et al., Testing modified gravity at cosmological distances with LISA standard sirens, JCAP 07 (2019) 024 [arXiv:1906.01593].

[55] M. Lagos, M. Fishbach, P. Landry and D. E. Holz, Standard sirens with a running Planck mass, Phys. Rev. D 99 (2019) 083504 [arXiv:1901.03321].

[56] R. C. Nunes, M. E. S. Alves and J. C. N. de Araujo, Forecast constraints on $f(T)$ gravity with gravitational waves from compact binary coalescences, Phys. Rev. D 100 (2019) 064012 [arXiv:1905.03237].

[57] R. C. Nunes, S. Pan and E. N. Saridakis, New observational constraints on $f(T)$ gravity through gravitational-wave astronomy, Phys. Rev. D 98, 104055 (2018) arXiv:1810.03942 [gr-qc].

[58] E. Belgacem, Y. Dirian, A. Finke, S. Foffa and M. Maggiore, Nonlocal gravity and gravitational-wave observations, JCAP 11 (2019) 022 [arXiv:1907.02047].

[59] R. D'Agostino and R. C. Nunes, Probing observational bounds on scalar-tensor theories from standard sirens, Phys. Rev. D 100 (2019) 044041 [arXiv:1907.05516].

[60] X. Gao and X. Y. Hong, Propagation of the gravitational waves in a cosmological background [arXiv:1906.07131].

[61] E. Belgacem et al., Cosmology and dark energy from joint gravitational wave-GRB observations, JCAP 1908 (2019) 015 [arXiv:1907.01487].

[62] G. Calcagni et al., Quantum gravity and gravitational-wave astronomy, JCAP 10 (2019) 012 [arXiv:1907.02489].

[63] C. Dalang, P. Fleury and L. Lombriser, Horndeski and the Sirens [arXiv:1912.06117].

[64] J. B. Jimenez, J. M. Ezquiaga and L. Heisenberg, Probing cosmological fields with gravitational wave oscillations [arXiv:1912.06104].

[65] W. J. Wolf and M. Lagos, Standard Sirens as a novel probe of dark energy [arXiv:1910.10580].

[66] M. Lagos and H. Zhu, Gravitational couplings in Chameleon models [arXiv:2003.01038].

[67] C. Dalang, P. Fleury and L. Lombriser, Horndeski and the Sirens [arXiv:1912.06117].

[68] E. Bellini and I. Sawicki, Maximal freedom at minimum cost: linear large-scale structure in general modifications of gravity, JCAP 1407 (2014) 050 [arXiv:1404.3713].

[69] J. Kennedy, L. Lombriser and A. Taylor, Reconstructing Horndeski theories from phenomenological modified gravity and dark energy models on cosmological scales, Phys. Rev. D 98 (2018) 044051 [arXiv:1804.04582].

[70] M. Denissenya and E. V. Linder, Gravity's islands: parametrizing Horndeski stability, JCAP 1811 (2018) 010 [arXiv:1808.00013]. 
[71] B. F. Schutz, Determining the Hubble constant from gravitational wave observations, Nature 323 (1986) 310.

[72] D. E. Holz and S. A. Hughes, Using Gravitational-Wave Standard Sirens, Astrophys. J. 629 (2005) 15 [astro-ph/0504616].

[73] H. Abedi, S. Capozziello, R. D'Agostino and O. Luongo, Effective gravitational coupling in modified teleparallel theories, Phys. Rev. D 97 (2018) 084008 [arXiv:1803.07171].

[74] R. D'Agostino and O. Luongo, Growth of matter perturbations in nonminimal teleparallel dark energy, Phys. Rev. D 98 (2018) 124013 [arXiv:1807.10167].

[75] S. Capozziello, R. D'Agostino and O. Luongo, Extended gravity cosmography, Int. J. Mod. Phys. D 28 (2019) 1930016 [arXiv:1904.01427].

[76] C. Gordon, K. Land and A. Slosar, Cosmological Constraints from Type Ia Supernovae Peculiar Velocity Measurements, Phys. Rev. Lett. 99 (2007) 081301 [arXiv:0705.1718].

[77] E. Barausse, The evolution of massive black holes and their spins in their galactic hosts, Mon. Not. Roy. Astron. Soc. 423 (2012) 2533 [Erratum ibidem 440 (2014) 1295] [arXiv:1201.5888].

[78] W. H. Press, S. A. Teukolsky, W. T. Vetterling and B. P. Flannery, Numerical Recipes, Cambridge University Press (1994).

[79] J. Gleyzes, Parametrizing modified gravity for cosmological surveys, Phys. Rev. D 96 (2017) 063516 [arXiv:1705.04714].

[80] N. Frusciante, S. Peirone, S. Casas and N. A. Lima, Cosmology of surviving Horndeski theory: The road ahead, Phys. Rev. D 99 (2019) 063538 [arXiv:1810.10521].

[81] C. D. Kreisch and E. Komatsu, Cosmological constraints on Horndeski gravity in light of GW170817, JCAP 12 (2018) 030 [arXiv:1712.02710].

[82] D. Alonso, E. Bellini, P. G. Ferreira and M. Zumalacarregui, Observational future of cosmological scalar-tensor theories, Phys. Rev. D 95 (2017) 063502 [arXiv:1610.09290].

[83] D. Jeong and M. Kamionkowski, Gravitational waves, CMB polarization, and the Hubble tension [arXiv:1908.06100].

[84] E. Bellini, A. J. Cuesta, R. Jimenez and L. Verde, Constraints on deviations from $\Lambda$ CDM within Horndeski gravity, JCAP 1602 (2016) 053 [arXiv:1509.07816].

[85] N. Cornish, D. Blas and G. Nardini, Bounding the Speed of Gravity with Gravitational Wave Observations, Phys. Rev. Lett. 119 (2017) 161102 [arXiv:1707.06101].

[86] G. W. Horndeski, Second-order scalar-tensor field equations in a four-dimensional space, Int. J. Theor. Phys. 10 (1974) 363.

[87] C. Deffayet, X. Gao, D. A. Steer and G. Zahariade, From k-essence to generalized Galileons, Phys. Rev. D 84 (2011) 064039 [arXiv:1103.3260].

[88] T. Kobayashi, M. Yamaguchi and J. Yokoyama, Generalized G-Inflation: Inflation with the Most General Second-Order Field Equations, Prog. Theor. Phys. 126 (2011) 511 [arXiv:1105.5723].

[89] L. Amendola, Cosmology with nonminimal derivative couplings, Phys. Lett. B 301 (1993) 175 [gr-qc/9302010].

[90] C. Germani and A. Kehagias, New Model of Inflation with Nonminimal Derivative Coupling of Standard Model Higgs Boson to Gravity, Phys. Rev. Lett. 105 (2010) 011302 [arXiv:1003.2635].

[91] A. Nicolis, R. Rattazzi and E. Trincherini, Galileon as a local modification of gravity, Phys. Rev. D 79 (2009) 064036 [arXiv:0811.2197].

[92] C. Deffayet, G. E. Farese and A. Vikman, Covariant Galileon, Phys. Rev. D 79 (2009) 084003 [arXiv:0901.1314]. 